\title{
Chaos in a modified Hénon-Heiles system describing geodesics in gravitational waves
}

\author{
K. Veselý, J. Podolský* \\ Institute of Theoretical Physics, \\ Charles University, \\ V Holešovičkách 2, 18000 Prague 8, Czech Republic.
}

November 3, 2018

\begin{abstract}
A Hamiltonian system with a modified Hénon-Heiles potential is investigated. This describes the motion of free test particles in vacuum gravitational $p p$-wave spacetimes with both quadratic ('homogeneous') and cubic ('non-homogeneous') terms in the structural function. It is shown that, for energies above a certain value, the motion is chaotic in the sense that the boundaries separating the basins of possible escapes become fractal. Similarities and differences with the standard Hénon-Heiles and the monkey saddle systems are discussed. The box-counting dimension of the basin boundaries is also calculated.
\end{abstract}

PACS: $05.45+\mathrm{b} ; 04.20 \mathrm{Jb} ; 04.30$

Keywords: Chaotic motion; Gravitational waves; Hénon-Heiles system

${ }^{*}$ E-mail: podolsky@mbox.troja.mff.cuni.cz 


\section{Introduction}

Various open Hamiltonian systems in which unbounded orbits exhibit a chaotic behaviour have been studied (in particular in the context of chaotic scattering), see e.g. [1]-[5] and elsewhere. A standard method of investigation and description of these systems is to examine a typical set of initial conditions and observe which of the outcome possibilities is assigned to each initial data. The presence of fractal basin boundaries separating the possible escapes in the space of initial conditions is a suitable and invariant characterization of chaos [6]- [8].

Among the simplest Hamiltonians of this type are those with two degrees of freedom and polynomial potentials, in particular the famous Hénon-Heiles (HH) system [9] given by

$$
H=\frac{1}{2}\left(p_{x}^{2}+p_{y}^{2}\right)+V(x, y),
$$

where

$$
V=V_{H H}(x, y) \equiv \frac{1}{2}\left(x^{2}+y^{2}\right)+\frac{1}{3}\left(x^{3}-3 x y^{2}\right) .
$$

In the literature, however, this is usually studied at energies below the critical escape value $E_{c}=\frac{1}{6}$, for which motions are bounded. These are regular for lowest $E$ but become chaotic at higher energies still obeying $E<E_{c}$. Chaos is also present above $E_{c}$, where unbounded orbits choose between three possible distinct escapes to infinity (see, e.g. (2]). The corresponding fractal basin boundaries have recently been investigated in [10].

In this paper a system with a potential similar to the standard HH model (22) is presented and studied. This naturally arises in the context of test particle motion in plane-fronted gravitational waves with parallel rays ( $p p$-waves) [11. It is well-known that in the simplest (so called homogeneous) $p p$-waves the geodesic motion is regular but, as shown recently [12]-[14], it is chaotic in the non-homogeneous case. It is the purpose of this paper to discuss in detail a combined effect of both homogeneous and non-homogeneous gravitational-wave contributions. In section 2 the geodesic equations of motion in $p p$-wave spacetimes are presented in a suitable form in which the relation to Hamiltonian dynamics is established. Specific energy manifolds are then described and classified in section 3. The onset of chaos investigated by numerical simulation of orbits in an interesting medium energy region is described in section 4. Finally, in section 5 the box-counting dimension of the fractal basin boundaries is calculated. 


\section{Geodesics in gravitational $p p$-waves with both homo- geneous and non-homogeneous components}

The line element of the well-known class of gravitational $p p$-wave solutions [11 in vacuum can be written

$$
\mathrm{d} s^{2}=2 \mathrm{~d} \zeta \mathrm{d} \bar{\zeta}-2 \mathrm{~d} u \mathrm{~d} v-(f+\bar{f}) \mathrm{d} u^{2}
$$

where $f(u, \zeta)$ is an arbitrary complex function of the retarded time $u$ and the complex spatial coordinate $\zeta$. As shown in [12], [13], to determine the geodesic motion in these space-times it is crucial to solve the complex equation

$$
\ddot{\zeta}+\left(U^{2} / 2\right) \frac{\partial \bar{f}}{\partial \bar{\zeta}}=0,
$$

for $\zeta(\tau)$, where a dot denotes the derivative with respect to the normalized 'time' parameter $\tau$, and $U \equiv \dot{u}$ is a constant. (The remaining functions $u(\tau)$ and $v(\tau)$ can be found by subsequent integrations.) Introducing two real coordinates by $x \equiv \mathcal{R} e \zeta, y \equiv \mathcal{I} m \zeta$, the Eq. (雨) can be considered as a consequence of the Hamiltonian (1) with the potential $V(x, y, u)=\left(U^{2} / 2\right) \mathcal{R} e f$.

The space-time (3) with the function $f$ linear in $\zeta$ represents the trivial flat Minkowski universe. The quadratic function $f \sim \zeta^{2}$ describes homogeneous $p p$-waves (see [11 and references therein), in which the particle motions are integrable. Geodesics in non-homogeneous $p p$-waves given by $f \sim \zeta^{n}, n=3,4,5, \cdots$, have only recently been investigated [12], [13]. The corresponding polynomial potential $V(x, y) \sim \mathcal{R} e \zeta^{n}$ is called an ' $n$-saddle' (a 'monkey saddle' for $n=3)$. It has been found that motion in these gravitational waves with constant 'amplitude' are unbounded with $n$ possible escapes to $|\zeta| \rightarrow \infty$, and that the basin boundaries separating these outcomes are fractal. For non-constant amplitudes describing sandwich and impulsive gravitational waves a smearing of chaos is observed [14].

Now, it is natural and also physically interesting to investigate the combined effect of quadratic and higher polynomial terms in the function $f$. This is the main purpose of our work here. The simplest situation of this type occurs when $f$ has both quadratic and cubic terms,

$$
f(\zeta)=\alpha \zeta^{2}+\frac{2}{3} \beta \zeta^{3}
$$

where $\alpha$ and $\beta$ are real positive constants. With this, (幽 reduces to $\ddot{\zeta}+\left(\alpha \bar{\zeta}+\beta \bar{\zeta}^{2}\right) U^{2}=0$, in which the (non-trivial) constants $\alpha, \beta$ and $U$ can be removed by a simple transformation

$$
\zeta(\tau) \rightarrow \frac{\beta}{\alpha} \zeta\left(\frac{\tau}{U \sqrt{\alpha}}\right)
$$


In the real coordinates $x$ and $y$, the equations of motion then take the form

$$
\ddot{x}+x+x^{2}-y^{2}=0, \quad \ddot{y}-y-2 x y=0 .
$$

These equations can be considered to follow from the Hamiltonian (1) with the potential

$$
V_{m}(x, y)=\frac{1}{2}\left(x^{2}-y^{2}\right)+\frac{1}{3}\left(x^{3}-3 x y^{2}\right)
$$

which may be called a 'modified Hénon-Heiles potential' since this has almost the form of the standard Hénon-Heiles potential (2) with the exception of the negative sign of the $y^{2}$ term. However, as will be shown below, this 'small' difference results in a behaviour which is qualitatively different from that observed in the well-known $\mathrm{HH}$ system. Note that, from a

previous investigation of a generalized HH system $V_{g}(x, y)=\frac{1}{2}\left(A x^{2}+B y^{2}\right)+\frac{1}{3}\left(C x^{3}-3 D x y^{2}\right)$ 'with adjustable coefficients', it is known that the system (8) (corresponding to the choice $A=C=D=1, B=-1$ in $V_{g}$ ) is not integrable [15].

\section{Classification of possible orbits according to a con- served energy}

First, we shall investigate specific properties of the polynomial potential (8) and compare them with those of the standard HH potential (2) and the monkey saddle potential $V_{m s}(x, y)=\frac{1}{3}\left(x^{3}-3 x y^{2}\right)$, in which the quadratic terms are missing. There are two equilibrium saddle points of the potential $V_{m}$ at $(x, y)=(0,0)$ and $(-1,0)$. This is different from the $\mathrm{HH}$ potential (2) for which there is an elliptic point at the origin (in addition to three saddle points), and from the monkey saddle potential which has only one unstable equilibrium point (at the origin). The equipotentials $V_{m}(x, y)=E=$ const. of the potential (8) are drawn in Fig. 1. Let us now briefly comment on various possible situations shown in the figure:

- For $E<0$ the energy manifold $H=E$ consists of three disjoint components (separated by a 'forbidden' grey region, see Fig. 1), each of which contains a single exit to infinity. The similar behaviour is observed for negative energies also in the monkey saddle potential $V_{m s}$ and in the $\mathrm{HH}$ system.

- For $E=0$ the two components of the energy manifold join at $(0,0)$, the third one remains separated. 
- For $0<E<\frac{1}{6}$ the energy manifold consists of two disjoint components, one containing a single exit to infinity $(x \rightarrow-\infty)$, the second containing two distinct exits $(y \rightarrow$ $+\infty, y \rightarrow-\infty)$. This situation does not occur in the monkey saddle potential, in which all energy manifolds with $E>0$ are connected and contain three exits to infinity. The energy manifolds $0<E<\frac{1}{6}$ for the HH system consist of four disjoint components. One corresponds to bounded orbits and the remaining three are unbounded, each containing a single exit to infinity.

- For $E=\frac{1}{6}$ the two components of the energy manifold join at the point $(-1,0)$.

- For $E>\frac{1}{6}$ the energy manifold is connected and contains three exits to infinity in this case. The topology of energy manifolds is the same for both the $\mathrm{HH}$ and the monkey saddle systems.

The orbits in the negative energy region cannot choose between different exits, so that no basin boundaries are present. On the other hand, the high energy region $\left(E>\frac{1}{6}\right)$ provides three possible exits to infinity and numerical simulations show that the basin boundaries are fractal indicating the presence of chaos. These simulations give results very similar to those for the monkey saddle presented in [13], or for unbounded motions in the HH model [10]. This is not surprising as the equipotentials of both the potential (8) and the standard $\mathrm{HH}$ potential (2) are well approximated by the monkey saddle equipotentials $V_{m s}=E$ for sufficiently high energies.

Thus, the most interesting energy region to be considered here is given by the condition $0<E<\frac{1}{6}$. Since the motion for $E<0$ is not chaotic while it is chaotic in the high energy region, chaos has to appear somewhere between $E=0$ and $E=\frac{1}{6}$. This is similar to the standard HH model, where the low energy orbits above $E=0$ are regular while they become chaotic for higher energies still below $E=\frac{1}{6}$. Such behaviour is not present in the 'pure cubic' monkey saddle potential $V_{m s}$ in which one can clearly distinguish only two energy regions, negative and positive. The existence of the medium energy region is caused by the presence of the quadratic terms in the potentials (21) and (8). In the following section we shall concentrate on the behaviour of orbits in this interval.

\section{The onset of chaos in the medium energy region}

For an investigation of orbits in the medium energy region $0<E<\frac{1}{6}$ by a fractal method (which is useful for the characterization of a sensitive dependence on initial conditions in 
systems with unbounded orbits) it is necessary to choose a suitable typical set of initial data. Here we may concentrate on motion in the region with two possible exits to infinity, $y \rightarrow \pm \infty$ (the right white region in Fig. $1 \mathrm{~d}$, e, f). Let us consider orbits starting at $\tau=0$ from rest from the 'right branch' of the level curve $B_{r}$ (the branch of $V_{m}(x, y)=E$ with $x>0$ ), see Fig. 2 for typical trajectories of this type. This choice of initial conditions is also useful for localizing periodic orbits, which play an essential role in establishing the chaotic behaviour in both the monkey-saddle system and the standard HH system above the critical energy [1], [2]. We can conveniently parametrize the starting points on $B_{r}$ using the polar coordinate angle $\phi$ with respect to the origin (such that $|\phi|<\frac{\pi}{6}$ ). Each orbit is then uniquely characterized by its energy $E$ and a starting angle $\phi$.

In fact, we can concentrate on a smaller range of $E$ in the medium energy region. It is easy to prove that for energies $0<E<\frac{1}{12}$ the orbits starting from $B_{r}$ cannot cross the $x$-axis. Indeed, the second equation of (17) can be rewritten as $\ddot{y}=y(1+2 x)$. Since $x>-\frac{1}{2}$ in the considered component of the energy manifold for $E<\frac{1}{12}$, the acceleration $\ddot{y}$ has the same sign as $y$. The basin boundary between the two possible exits ('up' and 'down') will therefore be given by $\phi=0$, as the orbits starting from $B_{r}$ with $\phi>0$ will always escape directly to $y \rightarrow \infty$, while the orbits with $\phi<0$ will always escape to $y \rightarrow-\infty$. They never cross the $x$-axis, so the basin boundary is not fractal. The unique orbit with $\phi=0$ is an unstable periodic orbit $\Pi_{0}$ with trajectory along the $x$-axis.

Consequently, chaos has to appear in the energy region $\frac{1}{12}<E<\frac{1}{6}$. Numerical simulations show that behaviour of orbits with small $\phi$ becomes much more complicated for energies $E$ above a certain critical value $E_{0} \in\left[\frac{1}{12}, \frac{1}{6}\right]$. The value of $E_{0}$ will be discussed and specified later. Typical orbits for $E=0.16$ are drawn in Fig. 2. In addition to the unstable periodic orbit $\Pi_{0}$ along the $x$-axis, there is an unstable periodic orbit $\Pi_{3}$ with $\phi>0$ (see the bold curves in Fig. 2). Another one $\Pi_{2}$ with $\phi<0$ (which is not shown) can be obtained by symmetry.

At this point it is convenient to introduce a simple notation. As mentioned above, there are two possible escapes to infinity for orbits in the region being discussed. Let us assign them a symbol $j$, where $j=2$ for the exit with $y \rightarrow-\infty$, and $j=3$ for the exit with $y \rightarrow \infty$ ( $j=1$ is reserved for the exit $x \rightarrow-\infty$, which is present for $E>\frac{1}{6}$, to keep the notation consistent with [13] and [14]). Also, let us denote by $\tau_{s}$ the time $\tau>0$ at which the orbit reaches infinity (we put $\tau_{s}=\infty$ for orbits that never escape to infinity).

We shall now investigate the detailed structure of the orbits for the typical energy $E=0.16$. In Fig. 3 the functions $j(\phi)$ and $\tau_{s}(\phi)$ are plotted. (These are analogues of 
the scattering function and the time delay, respectively.) The basin boundaries separating possible outcomes in the initial condition state space are clearly fractal, which is confirmed here by zooming in up to the fourth level. This is an invariant evidence that the behaviour of orbits for energy $E=0.16$ is chaotic. At each level of the fractal in Fig. 3, there are three peaks of $\tau_{s}(\phi)$. The left (middle, right) peak corresponds to orbits asymptotic (up to the given level) to the periodic orbit $\Pi_{2}\left(\Pi_{0}, \Pi_{3}\right)$. Observe that a fractal structure occurs in a region in which there are only two possible outcomes.

Further observations analogous to those described in [13], [14] could be made. Instead, we shall concentrate here on the feature which is not present in the monkey saddle system, namely the dependence of $j(\phi)$ on the energy $E$. In addition to a single-parameter $\phi$ dependence in Fig. 3, it is necessary to investigate the basins for the 'up' $(j=3)$ and 'down' $(j=2)$ exits in the two-parameter region $\left\{(E, \phi)\left|\frac{1}{12}<E<\frac{1}{6},\right| \phi \mid<\phi_{\max }=\frac{\pi}{6}\right\}$. The result for $|\phi|<0.1$ is presented in Fig. 4, in which black color denotes $j=3$ and white corresponds to $j=2$. As expected, the only basin boundary for $E$ below the critical energy $E_{0} \approx 0.13$ is the $x$-axis $(\phi=0)$. Above the critical energy this basin boundary bifurcates into three 'branches', the upper (with $\phi>0$ ) corresponding to the unstable periodic orbit $\Pi_{3}$, the middle corresponding to $\Pi_{0}$ and the lower corresponding to $\Pi_{2}$. Moreover, the basin boundaries above the critical energy $E_{0}$ are fractal. For example, the function $j(\phi)$ shown in the previous Fig. 3 (which is clearly fractal) can be obtained as a section of Fig. 4 for $E=0.16$. Fig. 4 is also suitable for a better localization of the critical energy $E_{0}$ (from Fig. 3 it was only clear that $E_{0}<0.16$ ). More detailed numerical calculations (not presented here) led us to the value $E_{0}=0.1364 \pm 0.0001$.

The chaotic part of the medium energy region $\left(E_{0}<E<\frac{1}{6}\right)$ in Fig. 4 contains two interesting subregions which deserve special attention: energies just below $E=\frac{1}{6}$ and energies just above $E=E_{0}$. These subregions will be discussed below.

Fig. 5 shows an enlargement of Fig. 4 for energies close to $E=\frac{1}{6}$. It is obvious that there is another bifurcation at $E_{1} \approx 0.165$ which is somewhat similar to that which occured at $E=E_{0}$. The fractal structure above $E_{1}$ becomes even more complex, roughly speaking with five peaks in $\tau_{s}(\phi)$ at each fractal level, instead of three peaks shown in Fig. 3.

However, Fig. 5 indicates more: as the energy $E$ approaches $\frac{1}{6}$, at least one other bifurcation occurs. This naturally leads us to the following conjecture: There is a infinite number of bifurcations in the fractal basin boundary below $E=\frac{1}{6}$ at each of which the basin boundary along $\phi=0$ bifurcates into three branches.

According to the conjecture, the density of bifurcation points grows to infinity as $E \rightarrow \frac{1}{6}$, 
where the third exit (to $x \rightarrow-\infty$ ) is opened. The periodic orbit $\Pi_{0}$ vanishes while a periodic

orbit $\Pi_{1}$ in the new 'neck' of the potential is created. Above $E=\frac{1}{6}$ there are three exits to infinity and three corresponding unstable periodic orbits $\Pi_{1}, \Pi_{2}, \Pi_{3}$, similar to the structure for the monkey-saddle system! (see [1], 13]).

It would be interesting to investigate the transition region around the first bifurcation point $E=E_{0}$ in detail. However, it proved to be very demanding on the accuracy of numerical methods. Fig. 6 indicates why: typical orbits in this energy region reach infinity at $\tau_{s} \gg 1$, after 'bouncing' many times between the potential walls ( $\tau_{s} \approx 400$ in Fig. 6 , which is still moderate: we were able to follow number of orbits with $\tau_{s}>10^{4}$ ). The orbit shown in Fig. 6 started at $\phi=0.002$ with $E=0.13669$ and performed 110 bounces before escaping to $j=2$. The numerical computation is extremely sensitive to the accuracy of the method. Such sensitivity is present close to any fractal basin boundary shown in Fig. 4, but it is usually restricted to a tiny range of initial conditions. However, it is present in the whole subregion bordered roughly by the rectangle $E_{0}<E<E_{0}+0.0005,|\phi|<0.003$. This region near the first bifurcation and similar (but smaller) regions near the higher bifurcations represent orbits with the maximum level of unpredictability in the system under consideration.

\section{The box-counting dimension}

Finally, we present a more complete characterization of the topology of chaotic orbits in the medium energy region. In discussion so far, we have restricted our analysis to specific (yet important) orbits starting from rest from $B_{r}$. A completely general orbit can start at an arbitrary (not forbidden) point with given energy $E$ and direction of the initial impulse. For our purposes it is crucial to investigate the character of the basin boundaries, i.e. their regular vs. fractal nature. All unbounded orbits starting at points $(x, y)$ with $y>0$ (or $y<0$ ) which never cross the manifold section $y=0$ has the same certain exit $j=3$ (or $j=2$, respectively). Therefore, the basin boundaries can appear only among those orbits which cross $y=0$. In order to obtain the basin boundary portrait it is thus sufficient to systematically study orbits which start from the (negative) $x$-axis, i.e. from points $P=(x \leq$ $0, y=0)$, where $x \in\left[x_{\min }(E), 0\right]$, with initial impulse $p_{x}, p_{y}$. (A complementary discussion of orbits starting at $x \geq 0, y=0$ would be analogous.) These orbits belong to the energy manifold $H=\frac{1}{2}\left(p_{x}^{2}+p_{y}^{2}\right)+V_{m}(x, 0)=E$. The set of initial conditions considered can thus be represented by a disc in the $\left(p_{x}, p_{y}\right)$ plane in which each point characterize a specific

\footnotetext{
${ }^{1}$ There are two other periodic orbits $\Pi_{4}$ and $\Pi_{5}$ in the monkey-saddle system, which are more difficult to localize. We do not discuss their analogues in our system.
} 
initial velocity $\dot{x}=p_{x}, \dot{y}=p_{y}$ of an orbit starting at $(x, 0)$, where $x \leq 0$ is the solution of the equation $x^{2}+\frac{2}{3} x^{3}=2 E-p_{x}^{2}-p_{y}^{2}$. The circular boundary of the disc corresponds to geodesics starting at $x=0$ with maximum permitted impulse $p_{x}^{2}+p_{y}^{2}=2 E$, whereas the center of the disc denotes $p_{x}=0=p_{y}$ and $x_{\min }(E) \in(-1,0)$. In Fig. 7 we present typical discs for $E=0.16$ (left) and $E=0.166$ (right) in which each point representing specific initial data has again been colored in black or white according to the exit ('up' and 'down', respectively) reached by the corresponding evolved orbit. Analogous discs in the monkey saddle potential can be found in [1], [13].

It is obvious that the discs contain fractal basin boundaries, patterns and bifurcations which resemble those shown in Fig. 4 and Fig. 5. Interestingly, the geometrical pictures are very similar despite the fact that they describe different situations. In Figs. 4 and 5 the main independent parameter is $E$, whereas $E$ is fixed in Fig. 7 with the main parameter being $x$.

The fractal structure of the basin boundaries can rigorously be characterized by the boxcounting dimension [3], [6]. A large number of randomly chosen initial conditions represented by points in the above discs is evolved. For each point $P$ its neighbourhood of radius $\varepsilon$ is also inspected. If not all the points in the neighbourhood belong to the same basin as $P$ then the point is denoted as 'uncertain'. The fraction $P(\varepsilon)$ of uncertain initial conditions among all chosen points (we simulated 10000 points for each $\varepsilon$ ) scales as $P(\varepsilon) \sim \varepsilon^{2-d}$, where the parameter $d$ gives the box-counting dimension of the fractal basin boundaries of the discs shown in Fig. 7. Numerical simulation indicates that $d=1.34 \pm 0.07$ for $E=0.16$, and $d=1.43 \pm 0.06$ for $E=0.166$. In fact, the dimension grows with $E$ from $d=1$ for $E<E_{0} \approx 0.1364$ to $d \rightarrow 1.50 \pm 0.05$ as $E \rightarrow \frac{1}{6}$.

\section{Concluding remarks}

We have discussed in detail the Hamiltonian system with the potential (8) which is a simple but interesting modification of the famous Hénon-Heiles potential (2). Interestingly, orbits in the system studied correspond to the geodesic motion of free test particles in the space-times describing plane-fronted gravitational waves with both homogeneous and non-homogeneous components in the structural function (5). We have demonstrated that for energies $E>$ $E_{c} \approx 0.1364$ the motion becomes chaotic since the basin boundaries separating two possible

exits to infinity have a fractal structure. For $E>\frac{1}{6}$ the third exit opens and the resulting chaotic behaviour has a similar structure as in the monkey saddle potential $V_{m s}$ described in [1], 13. This 'pure cubic' potential for the monkey saddle system is obtained by setting 
$\alpha=0$ in (5). On the other hand, if the cubic term in the function $f$ vanishes $(\beta=0)$, the gravitational wave is homogeneous and the equations of geodesic motion become linear, i.e. integrable.

\section{Acknowledgments}

We acknowledge the support of grants GACR-202/99/0261 from the Czech Republic and GAUK 141/2000 of the Charles University. We thank the developers of the software system FAMULUS which we used for computation and drawing of all the pictures. Also, we thank Jerry Griffiths for his help with the manuscript.

\section{References}

[1] D.L. Rod, J. Diff. Eq. 14 (1973) 129.

[2] R.C. Churchill, D.L. Rod, J. Diff. Eq. 37 (1980) 23.

[3] S. Bleher, C. Grebogi, E. Ott, R. Brown, Phys. Rev. A 38 (1988) 930.

[4] M. Ding, C. Grebogi, E. Ott, J.A. Yorke, Phys. Rev. A 42 (1990) 7025.

[5] E. Ott, T. Tél, Chaos 3 (1993) 417.

[6] E. Ott, Chaos in Dynamical Systems, Cambridge University Press, Cambridge, 1993.

[7] S.W. McDonald, C. Grebogi, E. Ott, J.A. Yorke, Physica 17D (1985) 125.

[8] C.P. Dettman, N.E. Frankel, N.J. Cornish, Phys. Rev. D 50 (1994) R618.

[9] M. Hénon, C. Heiles, Astron. J. 69 (1964) 73.

[10] A.P.S. de Moura, P.S. Letelier, Phys. Lett. A 256 (1999) 362.

[11] D. Kramer, H. Stephani, M.A.H. MacCallum, E. Herlt, Exact Solutions of Einstein's Field Equations, Cambridge University Press, Cambridge, 1980.

[12] J. Podolský, K. Veselý, Phys. Rev. D 58 (1998) 081501.

[13] J. Podolský, K. Veselý, Class. Quantum Grav. 15 (1998) 3505.

[14] J. Podolský, K. Veselý, Class. Quantum Grav. 16 (1999) 3599.

[15] M. Tabor, Chaos and Integrability in Nonlinear Dynamics, Wiley, New York, 1989. 


\section{Figure Captions}

Fig. 1 The equipotentials $V_{m}(x, y)=\frac{1}{2}\left(x^{2}-y^{2}\right)+\frac{1}{3}\left(x^{3}-3 x y^{2}\right)=E$ for nine values of energy $E$ representing all typical situations. The forbidden regions are grey.

Fig. 2 An example of orbits starting from rest from the 'right branch' of the level curve $B_{r}$ with energy $E=0.16$. The initial positions on $B_{r}$ are parametrized by an angle $\phi$. The orbits $\Pi_{0}$ and $\Pi_{3}$ are unstable periodic orbits.

Fig. 3 Plots of the function $j(\phi)$ labelling the two possible exits to infinity and the function $\tau_{s}(\phi)$ denoting the time at which the orbit starting at given $\phi$ reaches infinity $(E=0.16$ here). The enlargements up to the fourth level confirm the fractal structure of basin boundaries.

Fig. 4 The basins of the two exits, 'up' (black) and 'down' (white), in the subset of initial conditions corresponding to orbits starting from $B_{r}$. These are uniquely characterized by energy $E$ and starting angle $\phi$.

Fig. 5 Enlargement of Fig. 4 for energies close to $E=\frac{1}{6}$. The basin boundaries are fractal.

Fig. 6 Typical geodesics $(\phi=0.002, E=0.13669)$ 'bounce' many times between the walls before escaping to infinity.

Fig. 7 The discs $\left(p_{x}, p_{y}\right)$ for $E=0.16$ and $E=0.166$ show the topology of orbits in the corresponding energy manifold. Each point in the disc represents an orbit starting at specific point $x<0, y=0$ with a given velocity. Its colour denotes one of the two possible exits. Again, the fractal basin boundaries indicate chaos. 
This figure "Fig01.gif" is available in "gif" format from: http://arxiv.org/ps/gr-qc/0006066v1 
This figure "Fig02.gif" is available in "gif" format from: http://arxiv.org/ps/gr-qc/0006066v1 
This figure "Fig03.gif" is available in "gif" format from: http://arxiv.org/ps/gr-qc/0006066v1 
This figure "Fig04.gif" is available in "gif" format from: http://arxiv.org/ps/gr-qc/0006066v1 
This figure "Fig05.gif" is available in "gif" format from: http://arxiv.org/ps/gr-qc/0006066v1 
This figure "Fig06.gif" is available in "gif" format from: http://arxiv.org/ps/gr-qc/0006066v1 
This figure "Fig07.gif" is available in "gif" format from: http://arxiv.org/ps/gr-qc/0006066v1 